\title{
El derecho y la bioética
}

RAFAEL JUNQUERA DE ESTEFANI

Profesor titular de Filosofía del Derecho, UNED

No podemos defender que las mutuas relaciones e implicaciones que mantienen en la práctica la Bioética y el Bioderecho constituyan un tema de absoluta novedad. Si nos remontamos a los albores de la historia comprobaremos que Ética y Derecho o Moral y Derecho' han mantenido una variada gama de relaciones, yendo de la confusión total entre ambas a la separación radical. Pero, aún en este último caso sus mutuas conexiones se han mantenido inevitablemente. No parece extraño, por tanto, pensar que sus dos retoños, la Bioética y el Bioderecho, se verán implicados por esas relaciones.

A esta situación se le añade un nuevo dato: el avance biotecnológico de las últimas décadas. Dicho avance ha provocado que la reflexión moral se fijara en la incidencia de los nuevos descubrimientos biomédicos sobre el ser humano y su entorno, lo que a su vez ha supuesto un reflorecimiento de la Ética en un momento cuyo protagonismo se estaba diluyendo. Ese hecho de volver la vista hacia la biomedicina ha llevado, como es suficientemente sabido, a la necesidad de incorporar en esta reflexión a profesionales no sólo de la filosofía sino también del amplio es- 
pectro de las ciencias de la salud, de la naturaleza, de la teología, de la sociología, de la psicología, etc. También en esta reflexión multidisciplinar surge la necesidad de regular los posibles conflictos que van apareciendo y de proteger los intereses en juego, papel reservado en todo entramado social al Derecho. Esta confluencia entre la reflexión multi, pluri e interdisciplinar que supone la Bioética y la reflexión jurídica, así como la necesidad de una mutua colaboración y cooperación entre ambas es lo que va a constituir el objeto de nuestro estudio en este momento.

Para cumplir el objetivo propuesto seguiremos el siguiente iter: en primer lugar analizaremos la necesidad de la existencia del Bioderecho; en segundo lugar, veremos qué se entiende por Bioderecho; en tercer lugar, su necesaria relación con la Bioética; y en último lugar, el peligro de la otra vertiente resbaladiza.

\section{NECESIDAD DEL BIODERECHO}

La vocación del Derecho ha sido y es la de servir de instrumento para armonizar los diversos intereses que entran en confrontación en el variado sistema de las relaciones sociales. Pero, éstas no permanecen inalterables a lo largo de la evolución de la sociedad sino que cambian al mismo ritmo que ella. Este progreso de la realidad social ha originado que aparezcan nuevas situaciones donde las relaciones son más complejas y los intereses confrontados muestran una diversidad mayor. Así, ha sido una constante histórica que el Derecho ha tenido que afrontar estos cambios y adaptarse a las nuevas realidades.

Ahora bien, igualmente ha supuesto una característica de todo ordenamiento jurídico, como reconoce parte de la doctrina ${ }^{2}$, su poca capacidad de reacción ante esos cambios sociales. Esta falta de agilidad y flexibilidad le obligan a ir siempre a remolque de la evolución del grupo social. Ello se agudiza con la revolución biomédica y biotecnológica que 
se ha producido en los últimos tiempos, en los que el Derecho se ha visto totalmente superado por la situación e, incluso, retado por la propia sociedad para encontrar soluciones a los nuevos conflictos, armonizándolas con la necesidad de otorgar certeza y seguridad jurídica a los ciudadanos.

Ante esta situación el Derecho ha respondido tradicionalmente a través de tres vías ${ }^{3}$ : a) recoger las nuevas relaciones regulándolas normativamente o reconociéndolas jurisprudencialmente; b) aplicar a la nueva situación principios generales ya reconocidos jurídicamente (autonomía, dignidad humana, etc.); y c) adelantarse a la sociedad introduciendo nuevos valores mediante sus propios recursos, cosa que, normalmente, se realiza a través de la primera vía. Sin embargo, en el campo de la biomedicina "el objeto susceptible de tratamiento jurídico se distingue por su originalidad y por su no posible comparación con cualquier otra realidad social que el Derecho haya normado, además de requerir una delicadeza especial debido a que se trata de cuestiones en las que se entrelazan sentimientos muy íntimos del ser humano"4. No debemos perder de vista que, como mantiene el profesor RoMEo CASABONA ${ }^{5}$, aparecen nuevas perspectivas acerca de la comprensión por parte del ser humano de nociones fundamentales como son: la vida y la muerte; la salud y la integridad personal; la reproducción y la paternidad-maternidad; la dotación genética, etc. Y todas ellas confrontadas con la capacidad de decisión de los individuos, puesto que la nueva situación les posibilita para tomar decisiones sobre su propia vida, salud, etc., donde antes eran simples sujetos "pacientes" sometidos a la actividad benefactora de "otros" o dependientes de la misma naturaleza. Así, siguiendo a este autor, podemos decir que el problema jurídico consiste en descubrir cuáles son los nuevos valores ético-sociales necesarios para enfrentar la nueva realidad, cómo se van creando, y cómo integrarlos en el Derecho $^{6}$. Éste debe ser el papel a cumplir por el Bioderecho, para lo que estará en permanente relación con la Bioética, manteniendo ambos su propia independencia. 
Sin embargo, en sentido contrario algunos autores se oponen a la intervención del Derecho en este ámbito y a la existencia de una biolegislación específica, basados en diferentes razones ${ }^{7}$ (algunas de las cuales ya hemos adelantado) entre las que se encuentran las siguientes: a) la rapidez de cambio de la tecnología convierte en obsoletas las leyes nada más promulgarse, lo que obliga a una constante revisión y modificación de las mismas; b) las actuaciones científicas no conocen fronteras, mientras que las leyes sí, lo que produce movimientos migratorios buscando eludir el control normativo, siendo perjudicados los países más restrictivos; c) la legislación debe ser altamente técnica y provisional (por lo menos hasta que los riesgos queden sometidos a verificaciones), así, son los mismos expertos y técnicos relacionados con estas materias los que participarían en su redacción y en la toma de decisiones para controlar su propia actuación, con lo que estaríamos remitiéndonos a una autorregulación del científico; y, por último, d) la mayor parte de los peligros que se describen carecen de realismo. Como conclusión, esta postura defiende, en lugar de una regulación normativa, la existencia de unas directrices flexibles fundadas en la autoconciencia de una sociedad "libre y decente". Pero, ante semejante opción cabe plantear varios interrogantes: qué se entiende por dichas directrices, a quién se alude al hablar de "sociedad libre y decente" y si se refiere exclusivamente a un autocontrol por parte de los mismos científicos. Pero, estas posturas negacionistas no han conseguido tapar las voces que, desde el conglomerado social y desde la propia reflexión bioética, se han elevado pidiendo una actuación del Derecho.

El Bioderecho, por tanto, aparece para aportar a la Bioética conceptos e instituciones como autonomía, consentimiento ("informado"), capacidad, patrimonio, etc. Es una realidad que todos los ordenamientos jurídicos han venido exigiendo un mínimo de libertad a la hora de realizar determinados actos para que produjeran efectos en el mundo del Derecho. Igualmente, el hecho de otorgar el propio consentimiento ha sido considerado de vital importancia para la perfección de algunas ac- 
tuaciones jurídicas. Consentimiento que siempre se ha exigido que fuera informado y, por supuesto, libre (por lo que nos parece una reiteración hablar de "consentimiento informado"). No podemos olvidar un concepto como el de capacidad, que el Derecho ha distinguido en capacidad jurídica y capacidad de obrar y que han supuesto unas instituciones fundamentales de cara a la personalidad jurídica. Así, podríamos seguir hablando de otras muchas realidades que jurídicamente han sido construcciones doctrinales bien estructuradas y con siglos de elaboración y reflexión a sus espaldas, de tal forma que la Bioética es ahora cuando empieza a utilizarlas y no tiene más remedio que acudir al ámbito jurídico. De otra parte el Derecho también aporta toda su maquinaria normativa, reguladora y sancionadora, su sistema judicial, etc. Con estas afirmaciones queremos decir que el Derecho se ha convertido en un mecanismo muy útil para positivar la Bioética, para promocionar el cumplimiento de sus valoraciones a través de las normas jurídicas, de la coacción que las respalda y de los órganos que se encargan de su aplicación.

De igual manera, el Bioderecho ha tomado de la Bioética una nueva visión del concepto vida y todo lo que está relacionado con la misma (calidad de vida, salud, inicio de la vida, final de la vida, integridad física, etc.). También aporta o debe aportar al resto de disciplinas especializadas del Derecho una mayor flexibilidad y adaptabilidad a las situaciones emergentes y una definición más amplia y novedosa de determinadas instituciones jurídicas: familia, paternidad-maternidad, procreación, etc. Se constata que la nueva situación surgida como consecuencia de los avances biotecnológicos ha dejado obsoletas muchas de estas instituciones, obligando a su redefinición.

A esta situación se le añade una tendencia general a la juridificación de toda la sociedad. Por medio de ella el Derecho se ha convertido en un elemento básico ${ }^{8}$, dejando pocos "espacios libres", es decir no afectados por "lo jurídico", y la Bioética no puede ser una excepción. Sin embargo, aparecen algunos autores que ven este proceso no sólo como 
inevitable, sino también como imprescindible o necesario al darse en la Bioética las siguientes condiciones':

a. Sus juicios tienden a realizar distinciones, que resultan de vital importancia, muy desarrolladas entre lo que se puede y lo que no se puede hacer. Para proteger estos juicios uno de los mecanismos más útiles es el de la ponderación entre derechos y obligaciones, que es un mecanismo jurídico por naturaleza.

b. La actuación biomédica hace referencia a las buenas costumbres, a la moral, a los valores y normas, a las reglas y códigos deontológicos, etc., y, por ello, es necesario que se dé una reflexión serena basada en principios como el de buena fe o los recogidos en los derechos humanos, que también son juridicos.

c. En las relaciones médico-paciente cobran mayor fuerza términos como: garantías, procedimientos, obligaciones contractuales, responsabilidades, etc., que son jurídicos.

d. Ante los avances producidos en este campo se reivindica un derecho a la diferencia, a la diversidad, y al respeto a la dignidad del ser humano. Pero para ser efectivos necesitan dotarse de un sistema de garantías que es jurídico.

Por todos estos condicionantes y los expresados anteriormente, el Bioderecho se nos presenta como una rama del Derecho necesaria para completar la reflexión Bioética y para regular las relaciones y conflictos surgidos en el campo bioético. Una vez manifestada la necesidad de su existencia vamos a analizar qué es o debe ser el Bioderecho.

\section{CONCEPTO DE BIODERECHO}

En principio nos encontramos con que no existe unanimidad en el término a emplear: unos hablan de Biojurídica y otros de Bioderecho. Por nuestra parte, consideramos más correcto el segundo término puesto que no hablamos de "la Jurídica" sino del "Derecho". Así, no entendemos por 
qué cuando nos referimos a la parte de éste que tiene por objeto de regulación el campo biomédico tenemos que hablar de Biojurídica y no de Bioderecho. Es posible que se deba a un simple salto lingüístico: se pasa de hablar de "Bioética" a hablar de "Biojurídica". Sin embargo, no es el mismo caso, porque mientras que es correcto aplicar el término Bioética a una parte de la Ética, no lo es denominar Biojurídica a una parte del Derecho. Por ello nosotros hablaremos de Bioderecho.

El siguiente paso es clarificar el concepto de Bioderecho. En una primera definición podemos decir con la profesora MARCOS DEL CANO ${ }^{10}$ que se trataría de aquella parte del saber que se ocupa de analizar la incidencia de los fenómenos bioéticos en la ciencia del Derecho y la búsqueda del paradigma del Derecho capaz de normar estas situaciones de un modo acorde con las exigencias de dichos fenómenos ${ }^{\prime \prime}$. Dentro de este saber se incluirían como elementos del mismo: la biolegislación (conjunto de normas que regulan estas materias) y la biojurisprudencia (conjunto de decisiones judiciales referentes a los problemas que plantean las ciencias de la vida). Pero, estas dos parcelas no son más que partes de un todo constituido por el Bioderecho.

Para cumplir los objetivos que aparecen en esa definición, el Derecho debe hacerse eco del sentir de la sociedad y asumir aquellos valores que ésta vive como imprescindibles para conseguir su realización dentro del campo que nos ocupa. Asimilando esta función, el Bioderecho se fundamenta en dos pilares que sirven de cimiento a toda la reflexión jurídica y que constituyen los grandes valores inspiradores de cualquier sociedad moderna: la dignidad del ser humano y los derechos que le son inherentes. Partiendo de estos pilares se puede establecer el marco jurídico de actuación que respete la pluralidad de nuestras sociedades. Así, como expresa la profesora CASADo ${ }^{12}$, los Derechos Humanos están llamados a convertirse en el principal criterio regulador de las nuevas formas de control y de las nuevas posibilidades científicas y tecnológicas, propiciando y garantizando el respeto a la libertad, la igualdad y la dignidad de todos los seres humanos. 
Vistas de este modo las cosas, el Bioderecho ya cuenta en el ámbito universal con un marco delimitado por la Declaración Universal de Derechos del Hombre, así como los dos Pactos Internacionales de Derechos Económicos, Sociales y Culturales, y de Derechos Civiles y Políticos, la Declaración Universal sobre el Genoma y Derechos Humanos, y el Convenio de las Naciones Unidas sobre la Diversidad Biológica. En un nivel inferior podríamos continuar nuestra enumeración con las Declaraciones y Convenios regionales (Convenio relativo a los Derechos Humanos y la biomedicina del Consejo de Europa, por ejemplo), las diversas Constituciones de los países en las que quedan recogidos los principios básicos que deben regir las distintas sociedades ${ }^{13}$ y los derechos fundamentales de los ciudadanos, la legislación específica de cada Estado, etc.

Podemos concluir definiendo el Bioderecho como aquella parte del Derecho que tiene por objeto el estudio y regulación de las nuevas situaciones y relaciones biomédicas y biotecnológicas, desde el respeto a la dignidad y a los derechos del ser humano.

A continuación vamos a analizar la necesaria relación que existe y debe existir entre el Bioderecho y la Bioética.

\section{CORRELACIÓN ENTRE BIODERECHO Y BIOÉTICAl4}

Como ya hemos adelantado, la Bioética ha influido en el Bioderecho y éste en aquélla: ambos recaen sobre una misma realidad, ambos suponen códigos normativos, ambos se dirigen a los mismos destinatarios, por lo que no tienen más remedio que relacionarse. El Bioderecho necesitará que la Bioética le preste su reflexión ética multidisciplinar y la Bioética precisará de los instrumentos de aquél para hacerse efectiva y garantizar la armonía en las nuevas relaciones y situaciones sociales aparecidas.

Para un sector de la doctrina la juridificación de la Bioética no sólo se refiere a la mencionada positivación, sino que alude también al mé- 
todo jurídico de ponderar principios contrapuestos: para resolver determinados conflictos se procede a comparar los principios que están en juego estableciendo unas reglas de prioridad entre ellos que respondan a una argumentación racional ${ }^{15}$.

Este método se puede aplicar a los principios bioéticos ${ }^{16}$ que pretenden responder a cuatro cuestiones éticas ${ }^{17}$ : a- "¿quién debe decidir?"; b"¿qué daño y qué beneficio se puede causar?"; c- "¿cómo debe tratarse a un individuo en relación con los demás?"; y d- "¿qué se debe decir y a quién?". Pero, si analizamos estas cuestiones desde su ámbito más genérico y abstracto, nos daremos cuenta de que no son más que diversos aspectos de la cuestión ética central: ¿qué se debe hacer? Y su respuesta coincidirá con los cuatro principios derivados de las cuatro formulaciones del imperativo categórico de $\mathrm{Kant}^{18}$ : autonomía (nadie puede decidir por nosotros), dignidad (no se nos instrumentaliza), universalidad (no se nos trata peor que a los demás) y publicidad (conocer aquello que nos afecta para decidir). Ahora bien, estos principios sólo nos pueden ayudar a la hora de solucionar los llamados casos fáciles, pero son insuficientes para los conocidos como casos difíciles: ¿quién toma la decisión cuando el sujeto está incapacitado?, ¿qué ocurre si el paciente no quiere que se le dé toda la información?, ise respetarán las instrucciones que el individuo ha dejado para el caso de pérdida de la consciencia?, etc. La doctrina propone, para estos supuestos, unos principios secundarios derivados de aquéllos y son ${ }^{19}$ : a- el de paternalismo justificado (se pueden tomar decisiones que afectan a la vida o salud de otra persona en las siguientes condiciones: si ésta se encuentra en una situación de incompetencia básica, si supone un beneficio objetivo para ella y si se presume racionalmente que sería la decisión que tomaría dicha persona si gozara de plena consciencia); b- el de utilitarismo restringido (se puede realizar una actuación que no supone beneficio para el sujeto, en estos casos: si se obtiene un beneficio para otro u otros, si se cuenta con el consentimiento del afectado y si no se realiza ninguna acción degradante); c- el del trato diferenciado (se puede tratar a una persona de forma 
diferente a otra cuando concurren estas variables: si nos basamos en una circunstancia universalizable, si se produce un beneficio para otra $u$ otras y si se presume que el perjudicado consentiría en caso de encontrarse en unas situaciones de imparcialidad); y d- el del secreto (se pueden ocultar a las personas aquellas informaciones que afecten a su salud: si se trata de respetar su personalidad o si se hace posible una investigación a la que ha prestado su consentimiento). Sin embargo, los principios no van a poder resolver por sí mismos toda la diversidad de casos que pueden aparecer en la práctica bioética, se necesita acudir al mismo método que aplica el Derecho: precisar dichos principios en unas reglas. Éste es el problema fundamental de la Bioética para este sector de la doctrina: "pasar del nivel de los principios al de las reglas"20.

En nuestra opinión y coincidiendo con esta corriente, creemos que el paso de los principios a las reglas lo podemos dar a través de la juridificación de la Bioética, es decir, mediante el Derecho. Y éste puede llevarlo a cabo a través de un doble camino ${ }^{21}$ : el legislativo y el judicial. El primero tiene la ventaja de ofrecer mayor seguridad jurídica, puesto que queda establecido claramente en una norma escrita cuáles son las conductas que pueden realizarse y cuáles no, y eso se hace a través de los órganos elegidos democráticamente y que gozan, por lo tanto, de legitimidad. Hoy es muy frecuente que la sociedad demande del mundo jurídico una legislación que sustituya la falta de acuerdo social sobre estos asuntos, y ésta, realmente, es una de las funciones que debe cumplir el Derecho: la resolución de conflictos. Pero, aún así, se constata que las normas no dirimen de manera definitiva las cuestiones puesto que el debate social continúa y su aplicación puede dar lugar a nuevos conflictos $^{22}$. Ahora bien, esta primera vía se topa con el problema de tener que prever con antelación circunstancias y situaciones de una gran movilidad y variabilidad. Si tenemos en cuenta que una de las características del Derecho en su faceta legislativa es su poca capacidad de reacción ante los cambios sociales, comprenderemos por qué se ha visto superado una vez más por la nueva tipología de problemas y relaciones que 
han traído consigo las biotecnologías (nuevos modos de paternidad/maternidad creados por las Técnicas de Reproducción Humana Asistida, las posibles situaciones que genera la clonación, la manipulación genética, etc.) y la biomedicina (eutanasia, aparición de la medicina predictiva, nueva posición de los pacientes ante el médico, etc.). Por otro lado estas nuevas circunstancias requieren valoraciones o juicios morales ante los que es muy difícil obtener un consenso mínimo que recoja la opinión mayoritariamente expresada por la sociedad, por lo que esas normas corren el peligro de no responder a un acuerdo social, sino que se convertirían en una imposición de la valoración realizada por un determinado grupo que ostente el poder.

El segundo camino es el denominado judicial, no porque corresponda a los jueces, sino porque emplea el mismo método de ponderación de principios a través de unas reglas para resolver el caso concreto que se nos plantea ${ }^{23}$ y que hemos mencionado anteriormente. Esta vía puede recorrerse de varias formas. La primera, que nos imaginamos de inmediato, se realiza a través de los juzgados. Son los jueces los que toman las decisiones una vez que se les han planteado los casos, contando, para ello, con toda la mecánica jurídica y judicial que tienen en sus manos. Pero, nos volvemos a encontrar con la lentitud de la maquinaria del Derecho y su falta de elasticidad y flexibilidad para tomar decisiones rápidas y que respondan a las exigencias que las situaciones bioéticas plantean. La segunda forma de recorrer esta vía es a través de la actuación de Comités de Bioética (que han de contar con una composición interdisciplinar: juristas, éticos, biólogos, psicólogos, sociólogos, etc.) que siguiendo el método judicial, den respuesta rápida a los casos planteados. Dichos comités deben analizar los casos, deben tomar decisiones y elaborar unos informes. Se constata que estos organismos ya existen en diversos ámbitos ${ }^{24}$, realizando una labor de asesoramiento para los profesionales que acuden a ellos buscando criterios de decisión. Hoy, ya se ha comprobado la importancia y utilidad de estos órganos colegidos como medios de canalización de la creación de opinión, fomento del de- 
bate público y orientación de las instancias públicas ${ }^{25}$. Creemos, junto con el sector de la doctrina anteriormente citado ${ }^{26}$, que sería de gran interés la publicación de estas decisiones, así como su motivación y argumentación, creando una cuasi-jurisprudencia ${ }^{27}$ que puede servir de guía para futuras situaciones y casos que se presenten ante otros órganos o individuos que tienen que tomar decisiones en este campo.

Una vez analizados los caminos a través de los cuales puede ser juridificada la Bioética y vista la necesidad de relacionarse ambos campos, procede ahora concluir, siguiendo al profesor ROMEO CASABONA ${ }^{28}$, unos mínimos puntos de confluencia asumidos por esa dialéctica mutua que recojan lo desarrollado hasta este momento:

- La reflexión y búsqueda de principios regulativos o imposición de limitaciones no corresponde de modo exclusivo a los investigadores y científicos, puesto que las Ciencias Biomédicas comportan una incidencia directa sobre el ser humano y en su identidad colectiva. Por consiguiente el foro de debate ha de ser multi e interdisciplinar, lo que favorecerá que se tengan en cuenta todos los aspectos de la cuestión.

- Debe asegurarse en la discusión el pluralismo de todo tipo: ideológico, de creencias o concepciones de cualquier clase. Así, se garantizará que se integren todos los intereses enraizados en la sociedad.

- Como medios de canalización de la creación de opinión, fomento del debate público y orientación de las instancias públicas, se ha comprobado la importancia y utilidad de los Comités Nacionales de Bioética, independientes de los poderes públicos y de composición plural y diversa. Y, para la práctica cotidiana, también cumplen una función esencial los Comités hospitalarios.

- Se ha de tender a la uniformidad (dentro del pluralismo) de criterios tanto en el ámbito nacional como supranacional o internacional, armonizando las diversas legislaciones, evitando los "paraísos biotecnológicos". 
- El recurso a la regulación jurídica ha de reunir las siguientes características: a) debe ser prudente y sobrio, resolviendo exclusivamente los problemas inaplazablemente necesitados de regulación jurídica y sobre los que exista un mínimo acuerdo; b) debe ser elástico y abierto, no incompatible con otras concepciones y con nuevas perspectivas y situaciones; y c) debe ser variado, no basado exclusivamente en la norma legislativa, sino también en la actuación judicial, reconociéndosele al juez la capacidad de buscar soluciones creativas ante las nuevas situaciones, apelando a la actuación de Comités, etc.

Sobre estos mínimos, Bioética y Bioderecho pueden colaborar y aportar su reflexión, orientación y regulación a la sociedad ante los nuevos avances surgidos de la biotecnología.

Pero el Bioderecho también tiene su lado oscuro, sus peligros que vamos a analizar someramente a continuación.

\section{LA OTRA PENDIENTE RESBALADIZA}

En Bioética es muy conocido y utilizado el argumento llamado de la pendiente resbaladiza ${ }^{29}$ empleado para descalificar determinadas acciones porque, a pesar de ser buenas en sí mismas o al menos neutras, pueden desembocar en otra $u$ otras no deseables ${ }^{30}$. Se basa en un lenguaje metafórico expresando la situación de quien da un primer paso en una pendiente resbaladiza y ya no puede volver atrás o quedarse en ese punto, evitando caer hasta el fondo de la misma. Por lo tanto, si no queremos este desenlace deberemos eludir dar el primer paso ${ }^{31}$. Es un argumento bastante conservador que sirve para tomar posturas negativas en aras de posibles consecuencias que en un principio ni pueden imaginarse. En Bioderecho se utiliza para evitar las legislaciones excesivamente permisivas.

Pero, en este momento no se trata de realizar un estudio con detenimiento sobre esta argumentación, sino presentar el otro lado de la mis- 
ma. Puesto que estamos hablando en un lenguaje metafórico, vamos a pensar en una cadena montañosa con dos pendientes: la pendiente norte (Bioética) y la pendiente sur (Bioderecho). La pendiente norte es la argumentación tradicional en Bioética y que ha sido sucintamente explicada más arriba. La pendiente sur es la que vamos a denominar la otra pendiente resbaladiza en la que podemos caer al aplicar y utilizar el Bioderecho.

Cuando hacemos intervenir al Derecho en temas biomédicos y biotecnológicos, especialmente si utilizamos en exclusiva uno de sus instrumentos como es la legislación, estamos dando un primer paso en la pendiente jurídica para normativizar establemente situaciones, hechos, acciones, muy volubles y dinámicas. A este primer paso normativo, seguirá otro regulador de una nueva situación y así indefinidamente. Hemos iniciado el camino imparable de bajada, creando una sangría de normas (leyes, reglamentos, convenios, protocolos, códigos deontológicos, etc.) y utilizando la pesada maquinaria jurídica, difícilmente adaptables (ambos mecanismos) a los cambios que se vayan produciendo $y$, por lo general, presentando gran dificultad para su modificación. La sociedad irá cambiando con una velocidad de vértigo y nos encontraremos con un cuerpo normativo que estará vigente, pero que ya no responderá a las nuevas situaciones creadas por los avances biocientíficos. Nos veremos al final de la pendiente y con un cúmulo de normas cuya utilidad puede resultar escasa. De otra parte, es posible, que estas normas estén cargadas de prohibiciones con lo que supondrán una rémora para el avance y el progreso científico. Habrá que modificarlas e iniciar el camino jurídico para lograrlo. Ahora bien, esa maquinaria legislativa es muy lenta y compleja, lo que hará que el cambio de la normativa no se consiga fácilmente. Se comenzará el proceso y, mientras la sociedad y la técnica han variado, el Derecho y su regulación han sido incapaces de adaptarse ágilmente a ese cambio. Cuando la normatividad haya sido modificada, se encontrará con una situación que no es aquella a la que pretendía adaptarse y de nuevo nos veremos al final de la pendiente. 
Pero, esto no tiene por qué producirse si utilizamos el Derecho en sus dimensiones no exclusivamente normativas y punitivas. Creemos que es posible evitar esta nueva pendiente resbaladiza cuando el Bioderecho reúne las siguientes características:

- Que su regulación, como comentábamos anteriormente, sea: prudente y sobria; abierta y elástica; y variada; a estas últimas características ayudarían sobremanera dos elementos: la inclusión en cada norma de una cláusula, bien derogatoria o bien de revisión ${ }^{32}$ (son las normas conocidas en Derecho como normas de vigencia determinada) que obligara a que, una vez transcurrido cierto tiempo, se derogara dicha norma o se revisara potenciando su adecuación a los nuevos avances o descubrimientos, y la utilización de normas complementarias (decretos, protocolos, etc.) que colaboren en la regulación de aspectos muy concretos.

- Que no sea meramente prohibitivo. El Derecho puede utilizarse para configurar un marco de actuación de la ciencia donde se preserven sus intereses y los de la sociedad. Esto no sólo se consigue prohibiendo las actuaciones del científico, sino estableciendo unas condiciones donde se conjuguen la libertad de la ciencia y el bien y la seguridad de la sociedad.

- Que este Derecho se elabore utilizando los mecanismos de la llamada democracia deliberativa ${ }^{33}$, basada en la discusión abierta de los valores sustantivos que están en juego. Esto se realizará de la siguiente manera: cuando se va a proceder a regular las situaciones surgidas como consecuencia de los avances biomédicos, previamente se abrirá un proceso deliberativo en el que participen los diversos sujetos que representen los intereses que confluyen. En dicho proceso los diálogos se realizarán bajo las siguientes condiciones: la escucha atenta del parecer de los demás; la argumentación razonable para personas competentes en las respectivas materias; la tolerancia recíproca y el carácter provisional de las respuestas. En muchas ocasiones, al tratarse temas que afectan a toda 
la sociedad, deberá compulsarse el sentir global de la misma. De esta manera estaremos dando respuesta a lo que la colectividad requiere.

- Que se encuentre en constante conexión e interrelación con la Bioética. El Bioderecho no debe constituir una rama más del Derecho, sino que debe expresar una nueva forma de juridicidad que muestre una mayor flexibilidad y adaptabilidad y que esté en permanente diálogo con la Bioética ${ }^{34}$ que será quien le mantenga al día de los avances científicos y de la reflexión ética sobre los mismos.

- Que se otorgue un papel importante, entre todos los valores jurídicos (justicia, igualdad, libertad, paz social, orden público, etc.), a la solidaridad. Solidaridad con los enfermos de determinadas dolencias, con las generaciones futuras, con la especie humana en su totalidad, etc. No debemos ceñir nuestra visión a los intereses más inmediatos del científico o de la ciencia en concreto o de la sociedad actual. Tampoco debemos dejarnos cegar por los grandes valores "sacrosantos" y "desencarnados", sin contemplar los padecimientos de los enfermos. La solidaridad debe ensanchar el ángulo de nuestra visión y llevarnos a contemplar intereses difíciles de descubrir.

Cumplidos todos estos requisitos evitaremos caer por esta pendiente a la que nos arrastraría la inercia de la solemne y rígida maquinaria jurídica y podremos disponer de unas reglas de juego que favorezcan que la ciencia colabore en la consecución de un futuro esperanzador para el ser humano en las generaciones presentes y en las venideras. 
ABEL Y FABRE, F., Bioética: origenes, presente $y$ futuro, Madrid, Mapfre, 2000.

ATIENZA, M., "Juridificar la bioética", en VáZQQUE, R. (comp.), Bioética y derecho. Fundamentos y problemas actuales, México, Instituto Tecnológico Autónomo de México-Fondo de Cultura Económica, 1999, pp. 64-91.

Broekman, J. M., Bioética con rasgos jurídicas, (traducción del prof. Dr. H. Lindahl con la supervisión y coordinación de la Profesora Dra. M. Galán Juárez), Madrid, Dilex, 1998.

Casado, M., "Los Derechos Humanos como marco para el Bioderecho y la Bioética", en Romfo Casabona, C. M., (coord.) Derecho biomédico y bioética, Granada, Comares, 1998, pp. 113-135.

Casado, M., Bioética, Derecho y Sociedad, Madrid, Trotta, 1998.

ELIZARI, J., "El argumento de la pendiente resbaladiza", Moralia, n 4, 2001, pp. 469-490.

Gracia, D., Fundamentos de Bioética, Madrid, EUDEMA, 1989.
Hoff, P. F., Bioética y Derechos Humanos, Buenos Aires, De Palma, 1999.

JunqLera de Estéfand, R., "De Kant a Jonas: el principio de responsabilidad, Bioética y Derecho", en F. Llano (coord.) A propósito de Kant. Estudios conmemorativos del bicentenario de su muerte, Sevilla, Lagares, (se publicará en 2003).

Juyquera de Estéfani, R., Reproducción Asistida, Filosofia Etica y Filosofia Juridica, Madrid, Tecnos, 1998.

Marcos del Cano, A., "La biojurídica en España", Rivista Internazionale di filosofia del diritto, IV serie, LXXI, 1994, pp. 124-158.

Nossal, G. J. V., Los límites de la manipulación genética, (Traducción LÓPEZ, B.), Barcelona, Gedisa, 1997, segunda edición.

Romeo CasaboNa, C. M., "La relación entre la Bioética y el Derecho", en Romeo Casabona, C. M., (coord.) Derecho biomédico y bioética, Granada, Comares, 1998, pp. 151-164. 
1 No es este el momento ni el lugar de profundizar en las posibles diferencias entre moral y ética, así hablaremos indistintamente de una u otra.

2 Marcos del. Cano, A., "La biojuridica en Espan̄a", Rivisia Internazionale di filosofia del diritto, IV serie, LXXI, 1994, p. 126.

3 Romeo Casaibona, C. M., "Lá relación entre la Bioética y el Derecho", en Romeo Casabona, C. M., (coord,) Derecho biomédico y bioética, Granada, Comares, 1998, p. 15].

4 Marcos del. Cano, A., "La biojurídica...", cit., p. 125.

5 Romeo Casabona, C. M., "La relación entre la Bioética y...., cit., p. 152.

6 Ibídem, cit., p. 152.

7 Nossal, G. J. V., Los límites de la manipulación genética, (Traducción LÓPEZ, B.), Barcelona, Gedisa, 1997, segunda edición, pp. 158-160.

8 Brockman, J. M., Bioética con rasgos jurídicos, (traducción del prof. Dr. H. Lindahl con la supervisión y coordinación de la Profesora Dra. M. Galán Juárez), Madrid, Dilex, 1998, pp. 2830.

9 Ibídem, pp. 85-86.

10 Marcos del Cano, A., "La biojurídica...", cit., p. 132.

11 Una definición más compleja es la que da el profesor HoFFT: regulación jurídica en el plano de la alteridad de todas las cuestiones vinculadas con las ciencias de la vida, tanto en los planos de una micro, como meso o macro bioética, incluyendo las cuestiones referidas a la ecoética (HoFr, P. F., Bioética y Derechos Humanos, Buenos Aires, De Palma, 1999, p. 23).

12 Casado, M., "Los Derechos Humanos como marco para el Bioderecho y la Bioética", en Romeo Casabona, C. M., (coord.) Derecho biomédico y..., cit., pp. $118 \mathrm{ss.}$
13 En el caso de la Constitución Española, el art. 1.1 dice que los valores superiores del ordenamiento jurídico son la libertad, la justicia, la igualdad y el pluralismo político. Y el art. 10.1 declara que la dignidad de la persona, los derechos que le son inherentes, el libre desarrollo de la personalidad, el respeto a la ley y a los derechos de los demás son fundamento del orden político y de la paz social.

14 Algunos aspectos del contenido de este apartado se encuentran más desarrollados en JUNQUERA DE EstÉFAxI, R., "De Kant a Jonas: cl principio de responsabilidad, Bioética y Derecho", en F. Llano (coord.) A propósito de Kant. Estudios conmemorativos del bicentenario de su muerte, Sevilla, Lagares, se publicará en 2003.

15 ATIENZA, M., "Juridificar la bioética", en VázQuez, R. (comp.), Bioética y derecho. Fundamentos y problemas actuales, México, Instituto Tecnológico Autónomo de MéxicoFondo de Cultura Económica, 1999, pp. 73-82.

16 Los principios bioéticos ya clásicos desde el informe Belmont (EE.UU., 1978) y la obra de Benuchami, T. L., y childress, J. F., Principles of Biomedical Ethics son: autonomía, beneficencia, justicia y no maleficencia (este último fue añadido por los dos autores citados).

17 ATIENZA, M., "Juridificar la bioética", cit., p. 83.

18 Ibídem, p. 83.

19 Ibídem, pp. 86-87.

20 lbídem, p. 89.

21 Ibídem, p. 89.

22 CaSalo, M., Bioética, Derecho y Sociedad, Madrid, Trotta, 1998, p. 66.

23 ATIENZA, M., "Juridificar la bioética", cit., pp. 90-91.

24 Existen Comités de Centro en diversos hospitales, centros sanitarios o centros de 
investigación. Igualmente, existe la Comisión Nacional de Reproducción Asistida para asesorar al Ministerio de Sanidad en temas relacionados con la aplicación de las Técnicas de Reproducción Asistida (esta Comisión aparece creada por la Ley que regula estas Técnicas). Todos estos organismos tienen una función meramente asesora y no decisoria. Pero, en nuestro país no existe, como en otros de nuestro entorno, una Comisión de ámbito nacional y con competencias más genéricas que la que hemos citado. Creemos que sería de gran utilidad su creación y funcionamiento o bien seguir el criterio anteriormente citado de nucstra legislación y establecer distintas Comisiones nacionales que abarcaran diversos campos.

25 Romio casabona, C. M., "La relación entre la Bioética y el Derecho", en Romeo casabona, C. M (coord.), Derecho biomédico $y$ bioética, Granada, Comares, 1998, p. 162.

26 АTtE./A, M.. "Juridificar la bioética", cit., p. 91.

27 Hoy en día existen publicados informes de diversas comisiones o comités que han adquirido gran relevancia. Así podemos citar: Los dos informes anuales de la Comisión Nacional de Reproducción Humana Asistida, los informes del Observatori de Bioética i Dret (Barcelona), el informe del Comité de expertos sobre Bioética y Clonación del Instituto de Bioética de la Fundación de Ciencias de la Salud (Madrid), etc.

28 Romeo Casabona, C. M., "La relación entre la Bioćtica y..., cit, pp. 161-163.

29 También conocido como plano inclinado. En inglés slippery slope.

30 Para hacer un estudio más profundo de esta argumentación ver: ELIzARI, J., "BI argumento de la pendiente resbaladiza", Moralia, $n^{\circ} 4$, 2001, pp. 469-490.

31 Ibídem, pp. 470-472.

32 Es el caso contemplado en el artículo 32.4 del Convenio para la protección de los derechos humanos y la dignidad del ser humano con respecto a las aplicaciones de la Biología y la medicina (Convenio de Oviedo, del Consejo de Europa) en el que se dice: "con el fin de tener en cuenta los avances científicos, el presente Convenio será objeto de un estudio en el seno del Comité en un plazo máximo de cinco años a partir de su entrada en vigor, y en lo sucesivo, a intervalos que determinará el Comité".

33 A1311. Y FA13Rs, F., Bioética: origenes, presente y futuro, Madrid, Mapfre, 2000, pp. 212-215.

34 HoFrr, P. F., Bioética y..., cit., p. 23. 